\title{
Conservative Hepatic Hydatid Cyst Surgery
}

\author{
Mohamed Salah Abdelhamid ${ }^{1}$, Tamer Mohamed Nabil ${ }^{1}$, Hesham Ahmed Nafady ${ }^{1}$, \\ Amr Mohamed Ali ${ }^{1}$, Ahmed Mohamed Rashad ${ }^{1}$, Seham Anwar Emam ${ }^{1}$, Ibrahim Said Abdelaziz ${ }^{1}$, \\ Tamer Elgaabary ${ }^{2}$
}

${ }^{1}$ Surgery Department, Bani- Suef Faculty of Medicine, Bani - Suef University, Bani Suef, Egypt

${ }^{2}$ Surgery Department, Fayum Faculty of Medicine, Fayum University, Fayum, Egypt

\section{Email address:}

mohamedsalah_2000@hotmail.com (M. S. Abdelhamid), tamernbl@gmail.com (T. M. Nabil),

heshamahmednafady@yahoo.com (H. A. Nafady), Amr.bekheet@gmail.com (A. M. Ali), Rashad7793@yahoo.com (A. M. Rashad),

Siham.anwer8@gmail.com (S. A. Emam), Ibrahim sayed.@.yahoo.com (I. S. Abdelaziz), drtamerelgaabary@hotmail.com (T. Elgaabary)

\section{To cite this article:}

Mohamed Salah Abdelhamid, Tamer Mohamed Nabil, Hesham Ahmed Nafady, Amr Mohamed Ali, Ahmed Mohamed Rashad, Seham Anwar Emam, Ibrahim Said Abdelaziz, Tamer Elgaabary. Conservative Hepatic Hydatid Cyst Surgery. Journal of Surgery.

Vol. 5, No. 2, 2017, pp. 28-32. doi: 10.11648/j.js.20170502.14

Received: March 13, 2017; Accepted: March 23, 2017; Published: April 12, 2017

\begin{abstract}
The principal objectives to be achieved by surgical treatment are total removal of all parasitic elements, avoidance of spillage of contents of the cyst and management of the residual pericyst cavity. Thirty four patients with thirty five hepatic hydatid disease were included in the study. The data for this work were collected between 9-2015 till $9-2016$. The mean age were 38.6+13.2 years. There were twenty female and fourteen males. The main presentation was upper abdominal mass, 25 $(73.5 \%)$ were in the right lobe, $8(23.5 \%)$ were in the left lobe and only one $(3 \%)$ was bilaterally presented. Conservative surgery in the form of cystotomy (with or without omental packing), and external drainage were applied to 32 cysts $(91.4 \%)$ while radical surgery were applied to three cysts $(8.6 \%)$ in the form of pericystectomy and partial hepatectomy. LOS in the conservative group was $6.3+2.5$ day while in the radical group was $13.3+8.3$ day $(\mathrm{P}<0.001 \mathrm{HS})$. There were two cases of post operative bile leak in the omental pack group (22.2\%) and only one case (33.3\%) among the three cases of external drainage group, so in the conservative surgery there were three cases $(9.3 \%)$ and they resolved spontaneously. There were two redo one in the cystotomy patients, the other in the pericystectomy patients. Conclusion: Conservative surgery could be done with success in hepatic hydatid disease and resection procedures are too radical and extensive for benign lesions.
\end{abstract}

Keywords: Hepatic, Hydatid, Cysts, Unilocularis, Multilocularis, Conservative Surgery, Radical Surgery

\section{Introduction}

Hydatid disease remains an important health problem in endemic areas and by the way of travel and immigration it can be encountered in non endemic areas as well. Hydatid disease can occur in any viscera but affects most commonly the liver, and rupture of the liver cysts into the bile ducts during the evaluation of the disease is the most frequent complication [1].

Adult tapeworms of the genus Echinococcus are small and give their canine hosts little trouble, the intermediary hosts including man, bear the brunt of the disease. Man becomes the accidental host by ingesting canine tapeworm eggs, these hatch in the duodenum, releasing invasive embryos, which traverse the mucosa, enter portal circulation, and are swept first into the liver. After long periods of silent growth, large parasitic cysts-hydatids-are formed in the liver. Echinococcus granulosus, causing unilocular hydatid cysts, is the most prevalent species. Echinococcus multilocularis causing multilocular or alveolar hydatid cysts with unrestricted budding and without scolices invade the liver much like a malignant tumor invading the caval and portal veins. Echinococcus granulosus vogeli causes multilocular hydatid. The larvae lodge within the capillaries and first incite an inflammatory reaction composed of mononuclear cells. Many larvae are destroyed, but others encyst. The cyst begins at microscopic levels and progressively increases in size, enclosing an opalescent fluid is an inner nucleated germinal 
membrane, and an outer opaque non nucleated layer with innumerable laminations. Later minute projections of the germinative layer appear which develop central vesicles forming broad capsules (daughter cysts). Scolices of the worm develop on the inner aspect of these broad capsules and separate from the germinative layer to produce a fine, sand like sediment within the hydatid fluid [2].

Many surgical techniques have been used in the treatment of hydatid disease of the liver. The principal objectives to be achieved by surgical treatment are total removal of all parasitic elements, avoidance of spillage of contents of the cyst and management of the residual pericyst cavity [3]. In selecting a particular surgical technique, the surgeon should be guided by the size and location of the cyst and the complications it has already caused. Although several alternatives have been suggested for each step, selection of the most suitable approach depends on having a clear idea of the limitations as well as the long term consequences of all possible methods [4].

There are several ways of managing the residual cavity, depending on the contents of the cyst, location of the cyst and the preference of the surgeon. Some authors prefer to fill the residual cavity with sterile saline solution before water tight closure of the cavity, the idea being to decrease the possibility of an empty space, which may progress to a cavity filled with bile or localized hematoma. There is always, however the chance of secondary bacterial infection, which will not become absorbed for many weeks or months from the tightly closed space [5].

Marsupialization of the residual cavity has been tried repeatedly by some surgeons for many years, but inevitably, the results have been unsatisfactory. After removal of the initially placed drains, the cavity is found to have become a pool of purulent and bile-stained material. Leakage of bile from the cavity will persist for weeks and secondary sepsis is inevitable. The surrounding skin becomes inflamed, and the patient is left with a draining wound that takes months to obliterate. This procedure should be used only for infected cysts and in an occasional patient considered to be a poor risk [6].

Total cystectomy may be preferred for cysts located peripherally. Hepatic lobectomy and pericystectomy are too radical and extensive procedures for a benign lesion. Resection should be reserved for cysts caused by Echinococcus alveolaris only [7].

Internal obliteration by suture technique (capitonnage) carries the risk of injuring major ducts or vessels passing just outside the pericystic layer. More serious is the fact that the pericystic layer is stiff in long term cysts and walls of large pericystic cavities will just not come together. Either the sutures will tear out or a number of smaller loculations will remain [8].

According to some authors, after excising a reasonable portion of the redundant flaps of fused pericyst, the cavity may be left wide open (unroofing operation). However, this may result in intestinal obstruction, with a loop of intestine becoming stuck in the cavity. This cavity may be filled with omentum [9].

Introflexin is a simple and safe method. This technique not only prevent dead space, but also cover the inner surface of the cystic cavity by several layers of peritoneum, which has been demonstrated to have a high resorptive capacity [10].

\section{Patients and Methods}

The data for this work were collected between 9 -2015 till 9 -2016. Included in the study were 34 patients with hepatic hydatid disease. Their ages ranged from 6 years to 70 years with a mean+SD of $38.6+13.2$ years. There were twenty females and fourteen males. Among these thirty four patients there were two cases with recurrent hydatid, one operated upon elsewhere, the second was recurrent after our intervention.

\subsection{Clinical Features}

Abdominal pain was the main presenting feature. Most of the patients presented with abdominal discomfort, usually in the right hypochondrium, others in the epigastrium. In some an asymptomatic abdominal mass was discovered by chance with no previous complaint regarding the mass.

\subsection{Diagnosis}

Pre-operative diagnosis of a hydatid cyst was made from the clinical features with combination of investigations including ultrasound, computerized tomographic scanning and serology. Ultra-sound and CT scanning were accurate in locating the site, size and number of intrahepatic cysts, together with combined daughter cysts.

\subsection{Surgical Treatment}

The operative management was conservative. Exposure of the liver was obtained through a right subcostal incision. After exploration of the abdomen, the liver was mobilized and adhesions to the cyst divided. When firmly adherent to the diaphragm, particular care was required during the dissection to avoid entering the pleural cavity. The area around the cyst was then carefully isolated by gauze packs soaked in a scolicidal agent. Whenever possible, initial cyst aspiration and replacement with a scolicide was performed but this proved impossible with large cavities filled with daughter cysts. A trocar was used for evacuation, after which the edge of the collapsing cyst was grasped with tissue forceps and the sucker tubing itself inserted into the cavity to remove the numerous daughter cysts, the largest requiring removal by sponge-holding forceps. All laminated membrane was removed and the cavity carefully wiped with scolicidal soaked swabs. No attempt was made to remove the pericyst except from the surface of the liver. Sites of biliary leakage in the cyst wall were sutured. Whenever possible, a pedicled omental graft was fashioned and drawn up into the cavity to fill it loosely, the graft being held in position by catgut sutures. A drainage tube was laid alongside to provide egress for any biliary leakage. In most of the cases, the opening of 
the pericyst was sutured after filling the cavity with normal saline. Infected cysts are drained externally. All the patients are drained for at least three days and those who had bile leak, till two days after stopping the leak. All patients received pre-operative and post operative albendazole.

\section{Results}

Included in the study there were thirty four patients with thirty five hepatic hydatid cyst, as there was only one patient presented with bilateral hepatic hydatid. Only one extrahepatic hydatid cyst (retro-pancreatic cyst). There were twenty female and fourteen males, their ages ranged from 6 years and 70 years with a mean+SD of $38.6+13.2$ years.

There were twenty five patients with twenty five right hepatic lobe hydatid cysts, eight patients with eight left lobe hepatic hydatid, and only one patient with both left and right hepatic hydatid cysts (table 1). Thirty two were operated upon for the first time without previous attempt of surgical treatment, only they received medical treatment in the form of albendazole. There were only two recurrent cases, one operated upon elsewhere with right lobe cyst and an extrahepatic cyst (retro-pancreatic), the other one was recurrent after previous cystotomy, (two years after the $1^{\text {st }}$ intervention) and treated by pericystectomy.

Regarding segmental distribution (table 2), there was no cyst in segment I, two cysts $(5.7 \%)$ in both segment II \& III, while segment IV had 5 cysts $(14.2 \%)$. In the right lobe, segment $\mathrm{V}$ had two cysts $(5.7 \%)$, segment VI showed six cysts $(17.1 \%)$, segment VII was the highest incidence 10 cysts (28.5\%), segment VIII showed 8 cysts $(22.8 \%)$. There were only two cysts with cysto biliary communication (table 2 ).

Regarding the surgical techniques applied, there were twenty cases treated by simple cystotomy. Omental pack was used in nine cases There were three infected cases treated by external drainage. Two cases with recurrent cysts treated by pericystectomy. Only one case presented with peripheral left lobe medium sized cyst treated by partial hepatectomy (table 3).

The post operative hospital stay was $5.7+2$ days in cystotomy group of patients, $6.8+1.1$ day in omental packed patients, $22+2.6$ days in infected hydatid cysts with external drainage and 8 days in partial hepatectomy.

Regarding post operative bile leak, there were two cases in the omental packed patients group and one patient in the external drainage group. All of them stopped spontaneously on conservatism. In the cystotomy group, there was one patient re-admitted because of severe increasing upper abdominal pain with sub-phrenic collection, on exploration there was infected biliary collection, and the cyst was drained with the sub-phrenic space for almost three weeks. One patient in the pericystectomy was re-operated because of duodenal fistula (high out-put) following excision of the retro-pancreatic cyst and not related to the hepatic pericystectomy. Non of the patients who showed intraoperative cystobiliary communication had post operative bile leak.
Table 1. Distribution of hepatic hydatid cysts.

\begin{tabular}{lll}
\hline Site & No. of Patients & No. of Cysts \\
\hline Right lobe & $25(73.5 \%)$ & 25 \\
Left lobe & $8(23.5 \%)$ & 8 \\
Bilateral & $1(3 \%)$ & 2 \\
\hline
\end{tabular}

Table 2. Segmental distribution.

\begin{tabular}{llll}
\hline Segment & No. & Percent & Cystobiliary Communication \\
\hline I & - & & - \\
II & 2 & $5.7 \%$ & - \\
III & 2 & $5.7 \%$ & - \\
IV & 5 & $14.2 \%$ & 1 \\
V & 2 & $5.7 \%$ & - \\
VI & 6 & $17.1 \%$ & - \\
VII & 10 & $28.5 \%$ & 1 \\
VIII & 8 & $22.8 \%$ & - \\
\hline
\end{tabular}

Table 3. Surgical techniques.

\begin{tabular}{lll}
\hline Technique & No. & Indication \\
\hline Cystotomy & 20 & Ordinary cysts \\
Omental pack+partial pericystectomy & 9 & Huge cysts \\
External drainage & 3 & Infected \\
Pericystectomy & 2 & Rec. (Seg. VI - Seg. V) \\
Partial hepatectomy & 1 & Peripheral left cyst \\
\hline
\end{tabular}

Table 4. Post-operative follow-up.

\begin{tabular}{|c|c|c|c|}
\hline & LOS & & \\
\hline Technique & (mean+SD) & Bile Leak & Redo \\
\hline Cystotomy & $5.7+2$ & $1 / 20(5 \%)$ & $1 / 20$ \\
\hline Omental pack & $6.8+1.1$ & $2 / 9(22.2 \%)$ & $0 / 9$ \\
\hline External drainage & $22+2.6$ & $1 / 3(27.3 \%)$ & $0 / 3$ \\
\hline Pericystectomy & $16+9.8$ & $0 / 2$ & $1 / 2$ \\
\hline Partial hepatectomy & 8 & $0 / 1$ & $0 / 1$ \\
\hline
\end{tabular}

Table 5. LOS significance.

\begin{tabular}{lll}
\hline Technique & LOS & Statistics \\
\hline Conservative & $6.3+2.5 \mathrm{D}$ & $\mathrm{t}=3.8$ \\
Radical & $13.3+8.3 \mathrm{D}$ & $\mathrm{P}<0.001 \mathrm{HS}$ \\
\hline
\end{tabular}

\section{Discussion}

There is no clear consensus on the most ideal form of treatment of hydatid disease. Surgery should be the main stay of treatment for hydatid disease and that medical treatment should be restricted to patients not fit for surgery, or be used to prevent post operative complications. Liver hydatidosis cannot be considered a benign disease, as it is progressive and often recurrent $(10-15 \%$ of the cases). Mortality rates approach $10 \%$ in patients with recurrence. For these reasons surgery has remained the treatment of choice [11].

Right sided hepatic hydatid cysts were the commenest, as there were among the thirty four patients included in the study, twenty five $(73.5 \%)$ with right lobe cysts, followed by the left lobe, as there were eight patient $(23.5 \%)$. Only one patient presented by bilateral cysts (3\%). Wong et al [12] reported $70 \%$ right lobe, $17 \%$ left lobe and $13 \%$ both lobes. Segment VII had the highest incidence of affection 10 cases $(28.5 \%)$, followed by segment VIII and VI as there were eight $(22.8 \%)$ and six $(17.1 \%)$ cases respectively, then segment IV five cases $(14.2 \%)$, these findings are supported 
by many [4], [13] and [14].

There were two cases with recognized intra-operative cysto biliary communication and were closed by absorbable suture (vicryl 2/0), these two cases were in segment IV and VII and showed no post operative bile leak, so our incidence of cysto biliary communication was $5.8 \%$. Silva et al reported a very high incidence of such communication, they reported $50 \%$ which were over sewn using fine absorbable sutures. It is uncertain if these communications when present were spontaneous or introgenic [11]. Actually, incidence of cystobiliary communications depends largely on the criteria used for defining the communications. Kayaalp et al [4] was able to determine only $12 \%$ of the communications during intra-operative insistent search of cavities and this had increased the incidence. In histological studies of pericyst wall, numerous biliary ducts of various sizes communicated with the residual cavity were demonstrated [15]. Thus some authors claimed that these communications exist in almost all hydatid cysts [16].

Resection of the protruding dome exposes to specific longterm cavity-related complication, especially when hydatid cysts are located at the liver dome, while subadventitial cystectomy allows integrum restoration of the operated liver. Therefore, subadventitial cystectomy should be considered as the standard surgical treatment in experienced hepatopancreato-biliary centers [17].

Cyst size, stage, localization, and relation to the bile ducts are important in the treatment of liver hydatid cysts. The aim of surgical intervention is evacuation and obliteration of the cystic cavity. It is helpful to use ERCP or MRCP for the appropriate surgical method in patients with suspected communication with the biliary system. Uncomplicated cases of hepatic hydatid disease can be managed successfully by conservative surgery and the out come do not support the more wide spread use of hepatic resection or pericystectomy advocated by others [18].

Excision of giant hepatic hydatid cysts may be associated with loss of hepatic tissue. After simple cystectomy, the residual cavity is obliterated by manual compression of the healthy liver parenchyma from the left and right toward the midline of the cavity. While compression is maintained, the approximated edges of the cyst's fibrous capsule are closed with mattress sutures. Omentoplasty or gelatine sponges are used to fill the dead space prior to suturing of the fibrous capsule [19].

The treatment of liver echinococcal cysts represent a unique surgical challenge. Even though conservative approaches are less technically demanding, the radical approach with resection has better outcome with less recurrences, when performed by experienced surgeons. Resection rather than drainage is the management of choice for such huge liver echinococcal cysts. [20]

The results showed that most of the cases were uncomplicated isolated hepatic hydatid cysts frequently found on the right lobe of the liver. The most frequently implemented surgical procedure was partial cystectomy. This procedure was simple, fast and applicable for uncomplicated hepatic hydatid cysts [21].

We got four cases $(11.7 \%)$ of post operative bile leak, three of them recognized early $(8.8 \%)$ and one case $(2.9 \%)$ within two weeks after surgery. The two cases that showed intraoperative communications, showed no post operative bile leak. Two cases of early leak were in the omental pack group and stopped on conservative treatment and required no intervention, the leaks were moderate and the patients discharged from the hospital with the peritoneal drain with no prolongation of the hospital stay. The other (third case) was in the external drainage group and was marked but was decreasing progressively with prolongation of the hospital stay. The fourth case which was late was in the cystotomy group and manifested by cyst enlargement with sub-phrenic collection and necessetated redo to insert a sub-phrenic drain and another intra cystic drain with marked improvement after three weeks. Non of our patients required any form of biliary decompression or biliary exploration. There was no daughter cyst in the biliary tracts mostly due to the fact that almost all of our patients were unilocularis that is in contrast to multilocularis present in Turks, Kenea, East Europe that has the tendency to enlarge through perforation or invasion of the portal vein and portal tracts giving rise to distant metastatic cysts. Our finding goes hand in hand with Kayaalp et al [4] who stated that most of biliary leakage are self limited, as all of our biliary leakage stopped completely without any surgical procedure. Regarding redo there were two cases one due to the technique itself (cystotomy) as there was unrecognized cysto biliary communication and the other not related to the technique (pericystectomy) as the duodenal leak was not related to the main procedure but due to severe adhesion to the retro pancreatic hydatid cyst.

The conservative techniques applied (cystotomy - omental packing - external drainage) were very statisfactory regarding the LOS (mean+SD of $5.7+2 \quad 6.8+1.122+2.6$ ). Only in the external drainage group there was prolongation to gaurde against septic complications. Comparing this conservative technique to the more radical (pericystectomy partial hepatectomy) regarding $\operatorname{LOS}(6.3+2.5$ to $13.3+8.3)$ showed great difference in favour to conservative technique $(\mathrm{P}<0.001)$. Also the comparison with the less invasive approaches (percutaneous drainage) in favour with conservative technique as the prolonged contact between cyst wall in case of cysto biliary communication and the scolicidal agents used (alcohol - hypertonic saline) was associated with caustic sclerosing cholangitis [11]. We performed conservative surgery in most cases (32 cysts 94\%) like that of Kayaalp et al [4] who performed conservative surgery in $89 \%$ of their cases.

\section{Conclusion}

Conservative surgery could be done with success in hepatic hydatid disease and resection procedures are too radical and extensive for benign lesions. They were reserved only for cysts located peripherally and mostly pedunculated. 


\section{References}

[1] Alper A. Ariogul O, Emre A, et al. (1987): Choledechoduodenostomy for intrabiliary rupture of hydatid cysts liver. Br J Surg; 74: 243-5.

[2] Lucas SB (1994): Infectious diseases. In MacSween RNM, Anthony PP, Portman BC. Eds. Pathology of the liver, London: Churchill livingstone, $2^{\text {nd }}$ ed; pp 292-294.

[3] Danda MS, Guray M, Canda T, and Astarcioglu H. (2003): The pathology of echinococcosis and the current echinococcis problem in Western Turkey. Turk J Med Sci; 33: 369-374.

[4] Kayaalp C, Bostanci B, Yol S and Akoglu M. (2003): Distribution of hydatid cysts into the liver with reference to cytobiliary communications and cavity related complications. Am J Surg; 185: 175-179.

[5] Shirai Y, Wakai T, Ohtani T, et al. (1996): Colorectal carcinoma metastases to the liver, does primary tumor location affect its lobar distribution. Cancer; 77: 2213-6.

[6] Nap M, Hammerstrom M, Bormero, et al. (1992): Spedificity and affinity of monoclonal antibodies against carcinoembryonic antigen. Cancer Res; 52: 23-29-31.

[7] Dawson JL, Stamatakis JD, Stringer MD, and Williams R. (1988): Surgical treatment of hepatic hydatid disease. Br J Surg; 75: 946-50.

[8] Wigmore SJ, Madhavan K, Redhead DN, et al. (2000): Distribution of Colateral liver metastases in patients referred for hepatic resection. Cancer; 89: 285-7.

[9] Yilmaz E and Ozkok N. (1990): Hydatid disease of the liver: current surgical management. Br J Clin Pract; 44: 612-5.

[10] Langer JC, Rose DB, Keystone JS, et al. (1984): Diagnosis and management of hydatid disease of the liver. Ann Surg; 199: 412-7.
[11] Silva MA, Mirza DF, Bramhall SR, et al. (2004): Treatment of hydatid disease of the liver. Digestive Surgery; 21 (3): 227-34.

[12] Wong LS, Braghirolli No, Xu Min, et al. (1999): Hydatid liver disease as a cause of pancreatitis. JR Coll Surg Edinb; 44: 407-9.

[13] Fziri C, Paquet JC, Hay JM, et al. (1999): Omentoplasty in the prevention of deep abdominal complications after surgery for hydatid disease of the liver. J AM Coll Surg; 188: 281-9.

[14] Perdamo R, Alvarez C, Monti J, et al. (1997): Principles of the surgical approach in human liver cystic echinococcosis. Acta Tropica; 64: 109-22.

[15] Gahukamble DB, Khamage AS, Gahukamble LD (2000): Out come of minimal surgery for hydatid liver cyst. Ann Trop Ped; 20: $147-51$.

[16] Demirbag AE, Kayaalap C, Ozoguly, et al. (2000): Factors affecting biliary fistulae after hydatid surgery. Euro Surgery; 6: $20-24$.

[17] Mohkam K, Belkhir L, Wallon M, Darnis B, Peyron F, Ducerf C, Gigot JF, Mabrut JY. (2006): Surgical management of liver hydatid disease: subadventitial cystectomy versus resection of the protruding dome. World J Surg; 38 (8): 2113-21.

[18] Ali Ezer, Tarik Zafer Nursal, Gökhan Moray, Sedat Yildirim, Feza Karakayali, Turgut Noyan,. (2006): Surgical treatment of liver hydatid cysts. HPB (Oxford); 8 (1): 38-42.

[19] Julian E Losanoff, Bruce W Richman, James W Jones. (2004): Organ-sparing surgical treatment of giant hepatic hydatid cysts. AJS; 187 (2): 288-290.

[20] Pavlidis ET, Symeonidis N, Psarras K, Pavlidis TE. (2017): Huge echinococcal cyst of the liver managed by hepatectomy: Report of two cases Int J Surg Case Rep; 10 (31): 79-82.

[21] Yucel Y, Seker A, Eser I, Ozgonul A, Terzi A, Gozeneli O, Aydoga T, Uzunkoy A. (2015): Surgical treatment of hepatic hydatid cysts. A retrospective analysis of 425 patients. Annali Italiani di Chirurgia; 86: 437-443. 